\title{
Photospheric Flow Fields and Properties of Embedded Small-scale Magnetic Flux Concentrations
}

\author{
S. P. Rajaguru, R. Srikanth and S. S. Hasan \\ Indian Institute of Astrophysics, Bangalore- 34, INDIA
}

\begin{abstract}
The association between the different scales of convection on the solar photosphere and the field strengths/flux content of discrete magnetic flux concentrations is analyzed using simultaneously recorded SOHO/MDI high resolution filtergrams and magnetograms. The convective flow field is derived using the Local Correlation Tracking (LCT) technique. The locations and strengths of the flux elements with respect to the flow cells are analyzed to obtain information about different scales of convection.
\end{abstract}

\section{Convective cell and magnetic field organization}

An heirarchy of sizes in advecting flow patterns at the surface of Sun are known, viz. granules (0.7-1 Mm), mesogranules (3-10 Mm) and supergranules $(30 \mathrm{Mm})$. Of these, the convective nature of granules and supergranules (Leighton, Noyes, \& Simon 1962; Simon \& Weiss 1991) and many of their properties (sizes, life times) are well known. Convective nature of mesogranules is, however, a subject of much debate (Simon and Weiss 1991). Granules are the shallowest while supergranules possess the deepest downflows; a convective origin of mesogranules would imply an intermediate scale of downflow depth. The high plasma $\beta$ (i.e., weak magnetic fields) of the emerging photospheric magnetic fields, injected into the surface at the ephemeral active regions (Wang 1988) or obtained from the dispersal of dying active regions (Leighton et al. 1962), allows them to be swept to the edges of supergranules. Here opposing flows from neighboring cells confine the mixed polarity flux. Two key observations regarding flux accumulated at the edges of convective cells via flux expulsion: 1)the observed field strengths exceed the equipartition value $(500 \mathrm{G}), 2$ )the excess is observed mainly in supergranular edges. The plausible reasons (correspondingly): 1) convective collapsethe radial compression of flux into "fluxtubes" following evacuation of plasma in the downflow (Parker 1978). 2) Non-occurance of collapse due to (a) upward advection of granules in supergranular flow, and (b) the short lifetimes of granules which prevents sufficient collection of flux at their boundaries. Mesogranular lifetimes $(1-3$ hour) are long enough to invalidate (b); and if they are truely convective and form downflows to depths of the order of their size $\sim 7 \mathrm{Mm}$, then (a) too is not valid. Therefore, absence of observed strong fields at mesogranular edges may argue against convective origin of mesogranules. 

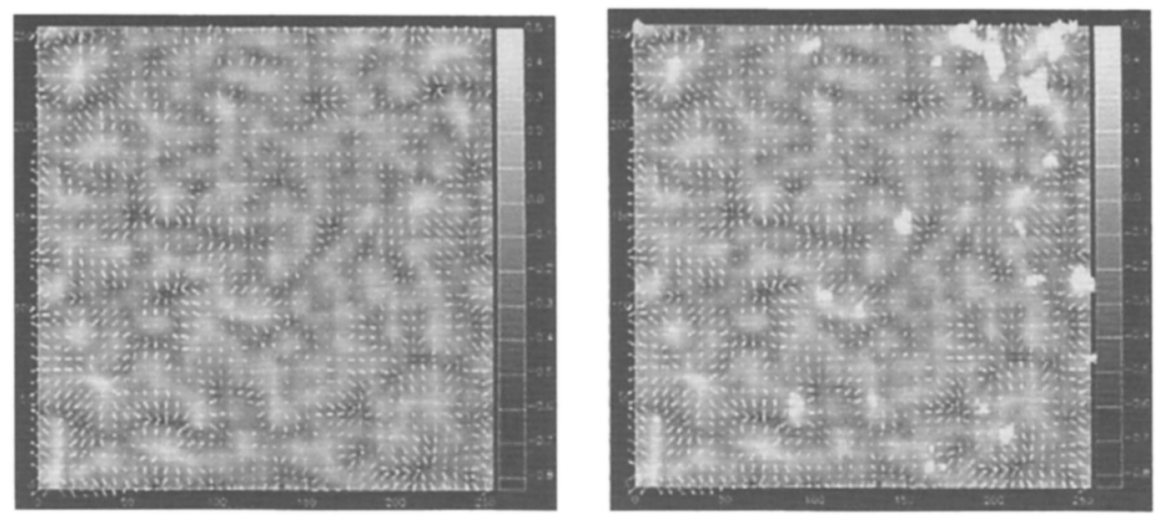

Figure 1. Left panel, 2-d flow divergence overlaid by granular flow field (arrows) derived from 2 hours of averaging; right panel, the same with the identified strong magnetic features marked by crosses. The axes are in pixels; 1 pixel $=0 .{ }^{\prime \prime} 605$

\section{Methodology}

We use SOHO/MDI high-resolution filtergrams and magnetograms to determine flow fields via LCT and positions and flux contents of discrete magnetic flux concentrations, respectively. These data-sets have $0 . " 605$ or $0.42 \mathrm{Mm}$ per pixel resolution and cover at most three days with a cadence of 1 minute. All the above are co-spatial and simultaneous (July 16-19, 1999) covering a region close to the disk center and include one active region; we analyse a quiet region field of view. After the initial pre-processing of data (Hagenaar et al. 1999) we apply the LCT algorithm of November \& Simon (1988) with a sampling Gaussian window of FWHM $7^{\prime \prime}$; we have avoided interpolation in the correlation space by achieving sub-pixel shifts in $x$ and $y$ directions before forming product maps and convolving with the apodizing window. The LCT map is derived at each point as the vector field that maximizes the correlation in product field. We identify supergranular boundaries with long-lived convergence loci in the flow field of granules. Mesogranules are identified as divergences at sub-supergranular scale ( Muller et al 1992). Since collapsed magnetic flux tubes $(B=1500 \mathrm{G})$ contain a minimum of about $5 \times 10^{17} \mathrm{Mx}$ flux (Rajaguru \& Hasan 2000), a pixel containing an unresolved flux tube has strength of about $250 \mathrm{G}$. This value is used as a minimum criterion to detect flux tubes. By comparing the LCT flow map and strong field positions, mesogranular boundaries are checked for presence of flux tubes.

\section{Results and conclusions}

Fourier interpolation to achieve sub-resolution shifts before forming the product images is found to give good flow maps at low time averaging (about 10 mins). We find that the supergranular horizontal flow is maintained my upflows (divergences) at the so-called mesogranular scale; these divergences have 
life-times in excess of $1 \mathrm{hr}$. Opposing flows from neighbouring divergences are deflected towards the supergranular boundary without forming a flow convergence (downflow) of similar lifetime around them. This feature is evident in Figure 1 (leftpanel) which shows a map of flow divergence overlaid by granular flow (arrows) derived from 2 hours of averaging and shows that there is no closed-cell circulation at the meso-granular scale; instead, these meso-scale divergences represent the scale of principal upflows maintaining the supergranular horizontal flow.

We find cases of substantial amount of magnetic flux presence in the centres of flow divergences, indicating emergence of flux from below. However, we do not detect any systematic correlation between the outflow boundaries (mesogranular edges) and strong field flux. Emerging flux from the upflow centres get advected away from them towards the supergranular boundary. In conjunction with the flow field scenario described in the previous paragraph, we explain the non-occurance of sustained flux presence surrounding flow divergences as that due to the absence of long-lived downflows around them. We are inclined to believe that mesogranules are not true convective cells (Ploner, Solanki, \& Gadun 2000). We confirm the presence of strong field flux at flow convergences in the supergranular boundaries, forming the so-called network. A movie showing the evolution of flow divergences (and convergences) and strong field flux concentrations has been made and is available at the URL http:// www. iiap.ernet.in/personnel/rajguru/index.html.

\section{Acknowledgments}

The SOHO/MDI data used in this work were provided by P. H. Scherrer and A. Kosovichev, Solar Oscillations Investigation (SOI), Stanford University, Stanford, USA. The first author S.P.R. is grateful to A. Kosovichev for encouragement.

\section{References}

Hagenaar, H.J., Schrijver, C.J., Title, A.M., Shine, R.A. 1999, ApJ, 511, 932

Leighton, R.B., Noyes, R.W., Simon, G.W. 1962, ApJ, 135, 474

Muller, R., Auffret, H., Roudier, T., Vigneau, J., Simon, G.W., Zoe Frank, Shine, R.A., Title, A.M. 1992, Nature, 356, 322

November, L.J., Simon, G.W. 1988, ApJ, 333, 427

Parker, E.N. 1978, ApJ, 221, 368

Ploner, S. R. O., Solanki, S. K., Gadun, A. S. 2000, A\&A, 356, 1050.

Rajaguru, S.P., Hasan, S.S. 2000, ApJ, 544 (to appear)

Simon, G.W., Weiss, N.O. 1991, MNRAS, 252, 1

Wang, H. 1988, Solar Physics, 116, 1. 